\title{
Gestão de Processos para o Gerenciamento de Redes de Circuitos Dinâmicos
}

\author{
Leonardo Carneiro, Sidney C. de Lucena, Flávia Maria Santoro
}

Programa de Pós-graduação em Informática - Universidade Federal do Estado do Rio de Janeiro (UNIRIO) - Rio de Janeiro - RJ - Brasil

leonardo.carneiro@rnp.br, \{sidney,flavia.santoro\}@uniriotec.br

\begin{abstract}
Dynamic Circuit Networks have been adopted by the foremost Research and Education Networks around the world. However, some management aspects of this kind of network remain unclear to the scientific community, characterizing it as a challenge to be overcome in the pursuit of providing a robust and reliable virtual circuit provisioning service. To address this issue, we propose the adoption of a business process management approach. The processes were adapted from conventional IP networks management processes, in which specific activities for dynamic virtual circuits were included. A case study was conducted and preliminary results evidenced that the adapted processes are applicable in a real Network Operation Center.
\end{abstract}

Resumo. As redes de circuitos dinâmicos vêm sendo adotadas pelas maiores redes acadêmicas do mundo. Porém, alguns aspectos da gestão deste tipo de rede ainda não estão claros para a comunidade científica, caracterizando-se como um desafio a ser superado na busca pela disponibilização de um serviço robusto e confiável de aprovisionamento de circuitos virtuais. Para resolver esta questão, propomos a adoção de uma abordagem de gestão de processos de negócios. Os processos foram adaptados de redes IP convencionais, nos quais atividades específicas para circuitos virtuais dinâmicos foram incluídas. Os resultados preliminares de um estudo de caso realizado evidenciaram que os processos adaptados são aplicáveis em ambientes de operação de redes.

\section{Introdução}

As chamadas National Research and Education Networks (NRENs) são redes de computadores de âmbito nacional, voltadas para o ensino e pesquisa, que interconectam os mais diversos institutos de ensino e pesquisa de um mesmo país, possibilitando trabalhos colaborativos entre os mesmos e a promoção do uso de novas tecnologias de redes. Como exemplos de NREN podem ser citadas a Internet2 [Internet2, 2013], a GÉANT2 [Géant2, 2013] e a RNP [RNP, 2013], sendo que a RNP é a instituição que provê e administra a rede de ensino e pesquisa brasileira.

Uma Rede de Circuitos Dinâmicos, ou Dynamic Circuit Network (DCN), tem como objetivo prover banda dedicada fim-a-fim para aplicações que demandem altas larguras de banda. Quando uma DCN coexiste com a mesma infraestrutura usada para uma rede IP tradicional, temos a Rede Híbrida. As DCNs, assim como seu uso em redes híbridas, são estudadas nas NRENs pela necessidade de atendimento a aplicações que demandam altas taxas de transmissão com requisitos rígidos de qualidade de serviço, 
tais como aplicações de telepresença, transferência de arquivos em grades computacionais, visualização de streams de vídeo com alta resolução etc.

No âmbito das NRENs, protótipos de redes híbridas estão em desenvolvimento para atender a estes serviços. Nestes protótipos, o serviço de aprovisionamento de circuitos virtuais é feito de forma dinâmica e automática através do uso de aplicações baseadas em Web Services, desenvolvidos por comunidades acadêmicas. Através destes sistemas, circuitos virtuais podem ser construídos dinamicamente, de forma independente das tecnologias e arquiteturas de rede disponíveis. Ou seja, o circuito virtual é estabelecido ao longo de uma infraestrutura de rede que pode ser composta por equipamentos de diversos tipos, como multiplexadores óticos DWDM, multiplexadores $\mathrm{PDH} / \mathrm{SDH}$, switches Ethernet, switches ATM e/ou roteadores IP/MPLS. Todos estes equipamentos são automaticamente configurados, sendo transparente, tanto para o usuário quanto para a aplicação, a infraestrutura sobre a qual o mesmo foi construído e a configuração aplicada nos equipamentos de rede.

Segundo [Kurose, 2006], o "gerenciamento de rede inclui a disponibilização, a integração e a coordenação de elementos de hardware, software e humanos, para monitorar, testar, consultar, analisar, avaliar e controlar recursos da rede, e de elementos, para satisfazer às exigências operacionais, de desempenho e de qualidade de serviço em tempo real a um custo razoável". Esta definição expõe a grande quantidade de parâmetros associados com o gerenciamento de redes mostrando a importância da busca por padronização. Dentro deste contexto, três órgãos internacionais se destacam como referência na área, e as discussões conduzidas resultaram nos modelos de gerenciamento de redes: FCAPS (Fault, Configuration, Accounting, Performance, Security) da ISO, TMN (Telecommunications Management Network) da ITU-T, e eTOM (enhanced Telecom Operations Map) do TMForum.

Contudo, nestes modelos, o gerenciamento das redes de circuitos virtuais dinâmicos ou não é abordado ou o é de forma restrita. Isto é, ao se tomar como base as áreas, processos e atividades de gerenciamento definidos nos modelos estudados, é possível verificar que tais conceitos não são aplicados no desenvolvimento de redes híbridas. Como resultado, observa-se que não existe, hoje, uma definição de como uma rede híbrida deve ser gerenciada, o que causa a falta de garantia de alta disponibilidade para este serviço, impactando diretamente a qualidade do serviço de rede.

O objetivo desta pesquisa é adaptar processos de gerenciamento de uma rede IP tradicional, tendo como base o modelo da ITU-T, de forma a definir processos e atividades operacionais que estruturem o gerenciamento de uma rede híbrida, contribuindo com uma nova abordagem baseada em processos. A aplicação da modelagem de processos de negócios em outros contextos pode ser vista em [Ostroski, 2012]. Como requisito, devem possuir o menor custo possível de adaptação de maneira a permitir o gerenciamento adequado deste novo ambiente de rede, sem ônus operacional ao gerenciamento do serviço de pacotes IP. Os processos operacionais desenhados para estruturar o gerenciamento da rede de pacotes da RNP, denominada rede Ipê, foram utilizados como base para as adaptações necessárias para contemplar o gerenciamento de redes híbridas. Desta forma, espera-se contribuir com processos de gerenciamento que proporcionem uma garantia de continuidade dos serviços de rede para a comunidade científica, seja de uma rede de pacotes tradicional ou de uma DCN. 
O artigo possui a seguinte estrutura: a Seção 2 apresenta conceitos básicos de redes híbridas e gerenciamento de redes; a Seção 3 discorre sobre modelagem de processos; a Seção 4 descreve os processos de gerenciamento adaptados para redes híbridas; a Seção 5 discute a avaliação da proposta; e a Seção 6 apresenta as conclusões.

\section{Gerenciamento de Redes e Redes Híbridas}

A ISO, entidade fundada em 1947, na Suíça, atualmente congrega os grêmios de padronização/normalização de mais de 170 países, aprovando normas internacionais em vários campos técnicos, propôs o modelo FCAPS. Este modelo resultou na Norma ISO 10040 e tem como principal objetivo classificar o gerenciamento de redes em grandes áreas de atuação: (i) Gerenciamento de Falhas, responsável pela detecção, isolamento, notificação e correção de falhas de rede; (ii) Gerenciamento de Configuração, responsável pelo registro e manutenção dos parâmetros de configuração dos serviços; (iii) Gerenciamento de Contabilidade, responsável pelo registro do uso da rede por parte de seus usuários, com objetivo de cobrança ou regulamentação de uso; (iv) Gerenciamento de Desempenho, responsável pela medição e disponibilização das informações sobre aspectos de desempenho dos serviços; e (v) Gerenciamento de Segurança, responsável por restringir o acesso à rede, protegê-la de atividades maliciosas e impedir o uso incorreto por parte dos seus usuários.

A ITU-T, comitê especializado da ONU, criado em 1947, é responsável por propor recomendações para o desenvolvimento de soluções tecnológicas envolvendo redes e telecomunicações. O Modelo TMN fornece um conjunto de funções que permitem realizar a gerência e a administração de uma rede de telecomunicações. $O$ modelo TMN introduziu o conceito de uma arquitetura lógica de gerenciamento baseada em camadas: gerenciamento de elementos, gerenciamento de redes, gerenciamento de serviços e gerenciamento de negócios. Em 1997, a ITU-T refinou o modelo FCAPS da ISO, integrando-o ao modelo TMN em sua Recomendação M.3400.

O TMForum, associação de empresas da indústria de telecomunicações, criada em 1988 e composta por mais de 700 empresas do setor, de mais de 185 países, tem como missão auxiliar as empresas prestadoras de serviços de telecomunicações na busca por uma maior eficiência na prestação dos seus serviços. O Modelo eTOM categoriza as atividades de negócio, definindo elementos-chave e a interação entre eles. O eTOM foi adotado como um padrão pela ITU-T em 2004 através da sua Recomendação M.3050.

Apesar de toda a importância atribuída ao gerenciamento de redes, não há uma proposta que trate de forma completa os requisitos para o gerenciamento de redes híbridas. Uma rede de circuitos dinâmicos (DCN) [Lake, 2008, Gudgin, 2007] combina comunicação de dados, por comutação de pacotes IP tradicionais, com tecnologias de comutação de circuitos características dos sistemas de telefonia tradicionais. Esta combinação provê ao usuário a alocação de uma largura de banda dedicada, de alta capacidade, que permite a execução de aplicações em tempo real e serviços de rede sobre uma infraestrutura de transmissão, podendo esta ser óptica [Naone, 2008]. Segundo [Lake, 2008], na arquitetura de uma DCN, recursos de rede, como largura de banda, são solicitados pelos seus usuários finais, automaticamente configurados por software de forma a prover circuitos virtuais e liberados quando os circuitos não são mais necessários. Adicionalmente, os recursos aprovisionados dinamicamente podem 
ser recursos de uma mesma rede, ou domínio, ou de redes diferentes. Isto é, domínios administrativos diferentes podem ter seus recursos aprovisionados de forma a prover um circuito virtual fim-a-fim.

Neste artigo propomos a abordagem da gestão de processos de negócios, especificamente a modelagem destes processos, como forma de definir processos e atividades que estruturem o gerenciamento de redes híbridas. Na seção seguinte, discutimos estes conceitos.

\section{Modelagem de Processos de Negócios}

Um processo de negócio, segundo [Cruz, 2003], classicamente é definido como sendo "o conjunto de atividades que tem por objetivo transformar insumos (entradas), adicionando-lhes valor por meio de procedimentos, em bens e serviços (saídas) que serão entregues e devem atender aos clientes". A gestão de processos de negócio inclui um conjunto de conceitos, métodos e tecnologias para suportar a análise, modelagem, configuração, execução e administração de processos de negócio [Weske, 2012].

A modelagem dos processos de negócio é uma das fases do ciclo de Gestão de Processos de Negócio. Ela tem sido adotada pelas organizações para suporte a melhores práticas e engenharia de seus sistemas de informação, além do conhecimento da forma como operam. A modelagem de processos de negócios é a atividade de representar processos de uma organização para que os mesmos possam ser analisados e melhorados, sendo seu produto o modelo de processos de negócio. No modelo são representados os elementos que constituem os processos de negócio, como atividades, eventos, informações, fluxos, atores e grupos. Além disso, para tratar a complexidade existente nestes modelos é aplicado o conceito de modularização ou decomposição funcional, que se refere ao mapeamento das funções organizacionais de forma hierárquica (top-down), detalhando o processo em níveis sucessivos (macro-processo, processo e sub-processo).

A modelagem de processos necessita de métodos e técnicas para auxiliar a organização na formalização de seu negócio. Nesta pesquisa, foi utilizada a técnica de fluxograma com uso da notação BPMN. Definiu-se a utilização de fluxogramas porque os processos originalmente desenhados para gerenciar redes IP convencionais, e sobre os quais foram propostas as adaptações, foram modelados com base nesta técnica.

\section{Processos de Gerenciamento de Redes Híbridas}

O macro-processo de Operações de Redes da RNP, para o qual foram propostas as adaptações para modelar o gerenciamento de redes híbridas, é o resultado do projeto de mesmo nome conduzido dentro do âmbito do Programa de Mudança Organizacional da RNP, que objetivou implantar o novo desenho organizacional da RNP. Neste contexto, foram produzidas as especificações de macro-processos, processos, sub-processos e atividades de Operações de Redes, assim como do inventário de competências e as demandas de sistemas de informação necessários para sua execução. O macro-processo de Operações, assim como os seus processos, sub-processos e atividades, foram desenhados pela equipe de analistas da Gerência de Operações da RNP com base nos modelos FCAPS e eTOM. O objetivo deste macro-processo é realizar a operação da infraestrutura da rede Ipê com excelência, apoiando o uso inovador de aplicações avançadas. A divisão hierárquica se deve à complexidade inerente aos processos de 
gerenciamento de redes e a possibilidade de maior aderência aos modelos de referência da ITU-T, pois foi possível trabalhar cada sub-processo desenhado como sendo uma das áreas de gerenciamento escritas nesta recomendação.

Os processos desenhados para atender à rede Ipê, uma rede IP convencional, partindo do macro-processo "Operações de Redes", são: "Preparação para a Operação" e "Operação e Manutenção de Redes". Estes dois processos, por sua vez, são divididos em oito sub-processos, conforme ilustrado na Figura 1. As adaptações propostas para contemplar o gerenciamento de redes híbridas são ressaltadas nas descrições dos processos, apresentadas a seguir. Dos oito sub-processos originalmente desenhados, não foram propostas alterações em três: "Gestão de Sistemas de Suporte à Operação"; "Gestão de Processos e Procedimentos"; e "Gestão da Segurança em redes". Contudo, o uso destes sub-processos na estruturação do gerenciamento de redes híbridas é válido.

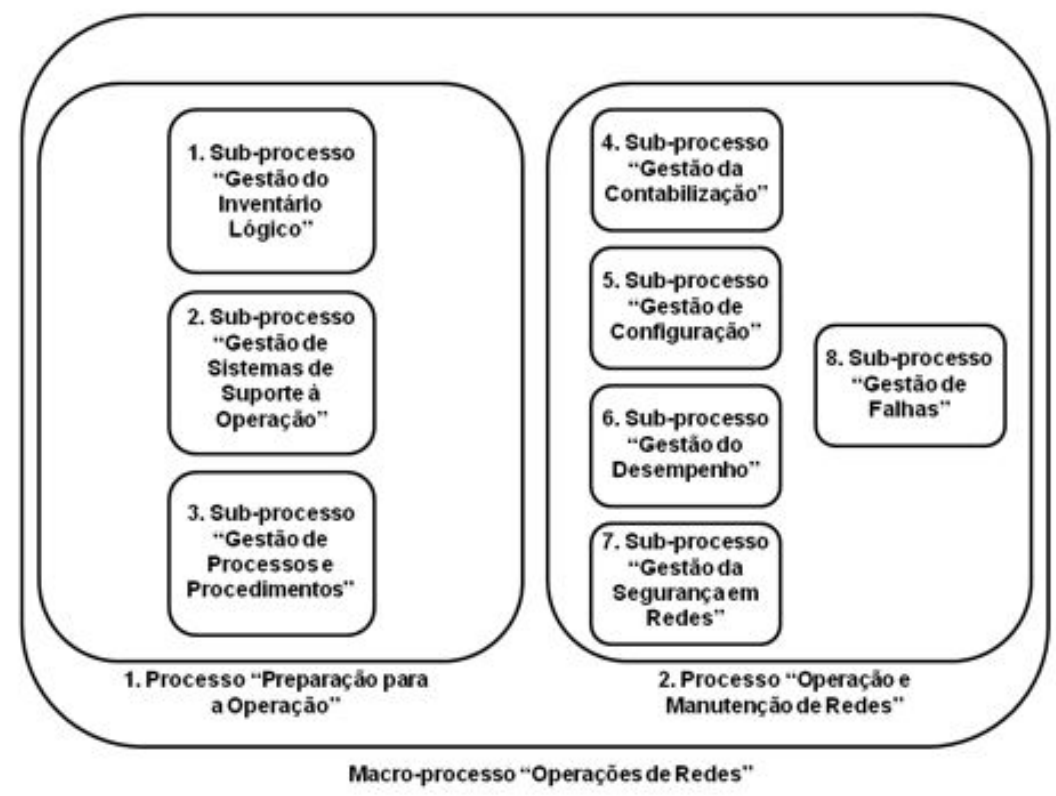

Figura 1. Processos do gerenciamento de rede IP convencional

\section{A. Sub-processo "Gestão do Inventário Lógico"}

Foi desenhado com base no grupo de processos funcionais horizontais RM\&O do modelo eTOM [M.3050.1, 2007], e inclui as seguintes atividades: (i) Manutenção de informações estáticas de circuitos, tanto de backbone quanto circuitos de instituições clientes da NREN; (ii) Manutenção de informações estáticas dos equipamentos de rede do backbone e dos clientes; (iii) Manutenção temporária de informações dinâmicas dos circuitos virtuais aprovisionados sob demanda pelos usuários da rede híbrida, onde recursos em questão podem ser: circuitos virtuais dinâmicos; (iv) banda alocada para o circuito virtual dinâmico; (v) início e fim da janela de tempo durante a qual o circuito virtual estabelecido dinamicamente deverá ser mantido; (vi) interfaces dos equipamentos de rede configurados automaticamente para o aprovisionamento do circuito virtual.

Adaptações propostas: (i) Inserção de demandas de aprovisionamento dinâmico de clientes, demandas que resultam em entradas que tratam ou do cadastro de um novo 
circuito virtual dinâmico de cliente ou da remoção do cadastro de um circuito virtual dinâmico de cliente. Esta adaptação foi proposta com base nas funções do processo Manage resource inventory, um dos processos que compõem o grupo horizontal funcional Resource Management and Operations (RM\&O) do modelo eTOM [M.3050.2, 2007]. (ii) Inclusão de operadores lógicos XOR no desenho do processo: para indicar que qualquer uma das entradas apresentadas pode disparar as referidas atividades, explicitando assim a decisão tomada, e a ligação dos fluxos de atividades desenhados na sua versão original, passando os mesmos a serem ligados pelas próprias saídas do sub-processo.

\section{B. Sub-processo "Gestão de Sistemas de Suporte à Operação"}

Foi desenhado com base no grupo de processos funcionais horizontais RM\&O do modelo eTOM [M.3050.1, 2007], e inclui as seguintes atividades: (i) Manutenção dos servidores utilizados internamente na área de Operações de Redes; (ii) Manutenção dos sistemas de acompanhamento de tíquetes; (iii) Manutenção dos sistemas de gerenciamento de rede que dão suporte à operação da mesma. Com relação ao aprovisionamento dinâmico de circuitos virtuais, o uso deste sub-processo é válido porque os softwares que permitem aos usuários da rede a construção dinâmica dos circuitos virtuais devem ser integrados aos softwares de gerenciamento da rede, acarretando na necessidade de potenciais ajustes nos softwares utilizados para aprovisionamento dos circuitos virtuais toda vez que as ferramentas de suporte à operação (OSS - Operations Support System) sofram alguma atualização.

\section{Sub-processo "Gestão de Processos e Procedimentos"}

Foi desenhado com base no grupo de processos Enterprise Effectiveness Management do modelo eTOM [M.3050.1, 2007], que aborda as seguintes atividades: (i) Atualização dos procedimentos internos da área de Operações de Redes; (ii) Avaliação da aplicabilidade dos procedimentos e processos; (iii) Atualização das políticas de uso da rede. Com relação ao aprovisionamento dinâmico de circuitos virtuais, o uso deste subprocesso é válido porque o mesmo tem a função de monitorar a eficiência e a efetividade dos outros sub-processos, podendo os mesmos serem alterados. Assim, quaisquer alterações podem acarretar em mudanças na forma como a rede híbrida será gerenciada.

\section{Sub-processo "Gestão da Contabilização"}

Foi desenhado com base nos conceitos de Accounting Management do modelo FCAPS, integrado ao modelo TMN [M.3400, 2000], e inclui as atividades de: (i) Coletar estatísticas de uso da rede; (ii) Armazenar estatísticas de uso da rede; (iii) Disponibilizar estatísticas para os clientes da rede; (iv) Analisar a utilização da rede com base nas estatísticas coletadas.

Adaptações propostas: (i) Inserção de demandas de aprovisionamento dinâmico de clientes que resultam em entradas que tratam ou do cadastro de um novo circuito virtual dinâmico de cliente, para coleta de estatísticas do seu uso, ou da remoção do cadastro de um circuito virtual dinâmico de cliente. Esta adaptação foi proposta com base nos conceitos de Accounting Management do modelo FCAPS, integrado ao modelo TMN [M.3400, 2000]. (ii) Inclusão de operador lógico XOR no desenho do processo para 
receber as diferentes entradas que disparam as referidas atividades, explicitando assim a decisão tomada, e a ligação dos fluxos de atividades desenhados na sua versão original, passando os mesmos a serem ligados pelas próprias saídas do sub-processo.

\section{E. Sub-processo "Gestão de Configuração"}

Foi desenhado com base nos conceitos de Configuration Management do modelo FCAPS, integrado ao modelo TMN [M.3400, 2000], e inclui as atividades de: (i) Coletar configurações feitas nos ativos da rede; (ii) Analisar comparativamente as configurações dos ativos da rede para checar configurações incorretas ou não planejadas; (iii) Remover configurações feitas nos ativos da rede.

Adaptações propostas: (i) Inserção de demandas de aprovisionamento dinâmico de clientes: demandas que resultam em entradas que tratam da configuração de equipamentos da rede para provimento de um novo circuito virtual dinâmico e da remoção das configurações de equipamentos da rede quando do fim do uso de um circuito virtual dinâmico. Esta adaptação foi proposta com base nos conceitos de Configuration Management do modelo FCAPS, integrado ao modelo TMN (M.3400, 2000). (ii) Inclusão de dois operadores lógicos XOR para receber as diferentes entradas que disparam as referidas atividades, explicitando assim a decisão tomada. (iii) Aplicação de gateway de decisão para contemplar as duas alternativas de saída.

\section{F. Sub-processo "Gestão do Desempenho"}

Foi desenhado com base nos conceitos de Performance Management do modelo FCAPS, integrado ao modelo TMN [M.3400, 2000], e inclui as atividades de: (i) Analisar o tráfego/utilização dos enlaces; (ii) Analisar erros em interfaces; (iii) Avaliar o trabalho de equipes terceirizadas; (iv) Avaliar os serviços prestados pelas operadoras; Avaliar os serviços prestados pelos fornecedores; (v) Realizar reuniões periódicas com equipes terceirizadas; (vi) Realizar reuniões periódicas com fornecedores; (vii) Avaliar Convênios. A Figura 2 ilustra as entradas deste sub-processo para tratamento de falhas recorrentes.

Adaptações propostas: (i) Inserção de gateway de decisão no fluxo de atividades para tratar de problemas recorrentes com o aprovisionamento dinâmico de circuitos virtuais. Esta adaptação foi proposta com base nos conceitos de Performance Management do modelo FCAPS, integrado ao modelo TMN [M.3400, 2000]. (ii) Inclusão de operadores lógicos XOR no desenho do processo para indicar que qualquer uma das entradas apresentadas pode disparar as referidas atividades, explicitando assim a decisão tomada, e definir um fluxo único de atividades.

\section{G. Sub-processo "Gestão de Falhas"}

Foi desenhado com base nos conceitos de Fault Management do modelo FCAPS, integrado ao modelo TMN [M.3400, 2000], e inclui as atividades de: (i) Acompanhar indisponibilidades (falhas em enlaces, ativos de rede, quedas de energia em PoPs, etc.); (ii) Acompanhar atualizações emergenciais em ativos de redes.

Adaptações proposta: (i) Inserção de gateway de decisão no desenho do processo para tratar de falhas ocorridas quando do aprovisionamento dinâmico de circuitos virtuais. Esta adaptação foi proposta com base nos conceitos de Fault Management do modelo 
FCAPS, integrado ao modelo TMN [M.3400, 2000]. (ii) Inclusão de operador lógico XOR no desenho do processo para concentrar todas as demandas do sub-processo em um fluxo único de atividades.

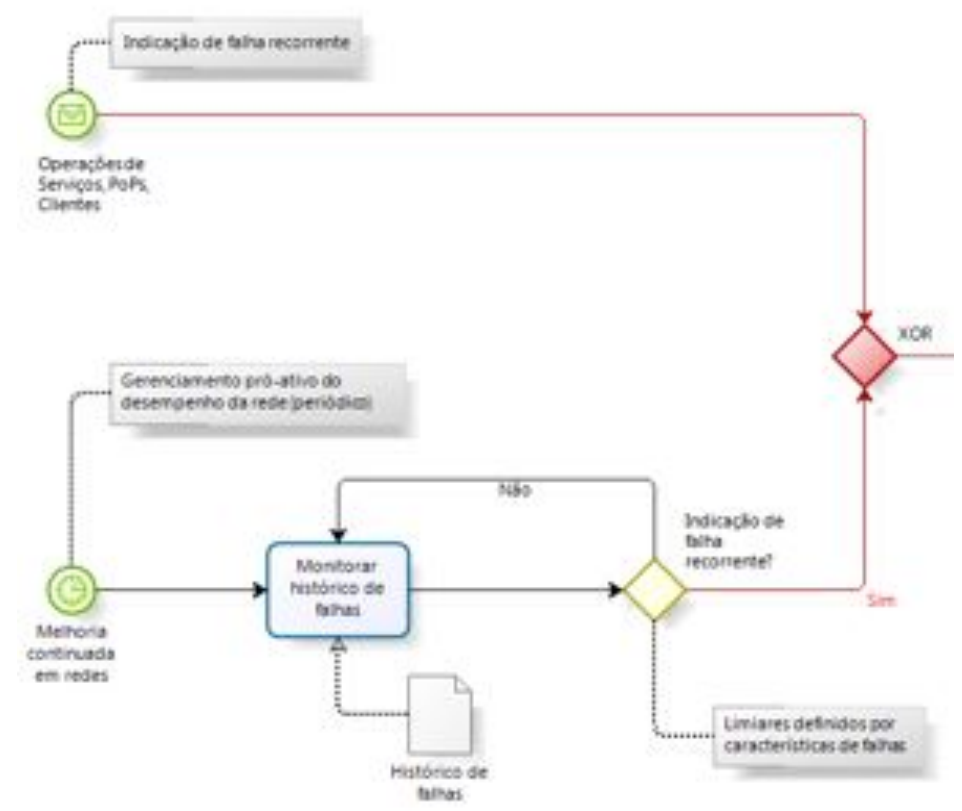

Figura 2. Entradas do sub-processo de gestão do desempenho.

\section{H. Sub-processo "Gestão da Segurança em Redes"}

Foi desenhado com base nos conceitos de Security Management do modelo FCAPS, integrado ao modelo TMN [M.3400, 2000], e inclui as atividades de: (i) Mitigar incidentes de segurança; (ii) Gerar relatórios de segurança. Com relação ao aprovisionamento dinâmico de circuitos virtuais, o uso deste sub-processo é válido porque os domínios de rede dos clientes que possuem softwares construtores de circuitos virtuais podem ser atacados e invadidos, ocasionando um incidente de segurança originado pelo mau-uso do serviço de aprovisionamento de circuitos.

A partir dos modelos de processos construídos, foram propostas indicações de como integrar as ferramentas de aprovisionamento de circuitos virtuais e de monitoramento, permitindo a elas operarem de forma integrada a ambientes reais de operações de redes, facilitando o entendimento dos operadores de rede sobre como monitorar um circuito virtual dinâmico. A Tabela 1 resume as indicações de integração.

Tabela 1: Indicações de integração de ferramentas de aprovisionamento com ferramentas de monitoramento

\begin{tabular}{|l|l|}
\hline Integração & Descrição \\
\hline $\begin{array}{l}\text { Cadastro/Atualização do } \\
\text { inventário lógico }\end{array}$ & $\begin{array}{l}\text { Uma vez que a ferramenta de aprovisionamento cria/apaga um circuito virtual, os seus parâmetros são automaticamente } \\
\text { cadastrados/removidos no/do inventário lógico (usado pelas ferramentas de monitoramento). }\end{array}$ \\
\hline $\begin{array}{l}\text { Cadastro/Remoção de } \\
\text { configurações }\end{array}$ & $\begin{array}{l}\text { Uma vez que a ferramenta de aprovisionamento cria/apaga um circuito virtual, ela automaticamente executa a ferramenta } \\
\text { de monitoramento responsável pela gestão de configuração, alarmando ao NOC as configurações aplicadas/removidas } \\
\text { na/da rede para construção do circuito virtual. }\end{array}$ \\
\hline $\begin{array}{l}\text { Coleta/Remoção de } \\
\text { estatísticas de uso }\end{array}$ & $\begin{array}{l}\text { Uma vez que a ferramenta de aprovisionamento cria/apaga um circuito virtual, ela automaticamente executa a ferramenta } \\
\text { de monitoramento responsável pela gestão de desempenho, iniciando/removendo a coleta das estatísticas de uso do circuito } \\
\text { virtual recém-criado/recém-apagado. }\end{array}$ \\
\hline Monitoramento de falhas & $\begin{array}{l}\text { Uma vez que a ferramenta de aprovisionamento cria/apaga um circuito virtual, ela automaticamente executa a ferramenta } \\
\text { de monitoramento responsável pela gestão de falhas, configurando/desconfigurando os parâmetros do circuito virtual } \\
\text { passíveis de monitoramento. }\end{array}$ \\
\hline
\end{tabular}




\section{Avaliação da Proposta e Resultados Obtidos}

O método aplicado foi avaliação por especialistas, uma vez que, sendo as redes híbridas hoje disponíveis apenas através de protótipos, os processos adaptados para contemplar o gerenciamento de circuitos virtuais dinâmicos ainda não foram aplicados em um ambiente real de operações. Desta forma, sua aplicabilidade e confiabilidade só podem ser inferidas com base na experiência dos especialistas. A avaliação dos processos foi planejada e realizada a partir da elaboração de dois documentos: uma apresentação sobre a pesquisa, na qual foram disponibilizados os processos de gerenciamento adaptados; e um questionário, formado por questões objetivas e dissertativas, no qual se buscou avaliar a aplicabilidade e a confiabilidade dos processos. Foram também realizados os seguintes passos: seleção de especialistas em redes que trabalham em ambientes reais de operações de redes; apresentação da pesquisa para os mesmos; aplicação do questionário aos especialistas selecionados; e análise das respostas dos especialistas, evidenciando as descobertas e conclusões.

A avaliação foi realizada na Rede Nacional de Ensino e Pesquisa, que é uma das NREN na qual o conceito de rede híbrida vem sendo estudado e um protótipo vem sendo desenvolvido [SE-CIPÓ, 2013]. Inicialmente, os processos foram apresentados em uma reunião presencial para os seis analistas da Gerência de Operações. Ao longo da apresentação, os especialistas tiveram a liberdade para expor sua visão sobre o tema e as suas impressões sobre as adaptações nos processos. A dinâmica foi aplicada com base na técnica Grupo Focal que, tem origem na técnica de entrevista em grupo, sendo utilizada em pesquisas qualitativas com o objetivo de coletar dados [De Antoni, 2001]. Conforme [Iervolino, 2001], Grupo Focal "pode ser utilizado no entendimento das diferentes percepções (...) acerca de um (...) produto ou serviço".

Ao fim da apresentação, foi encaminhado para os analistas o questionário de avaliação dos processos. A apresentação feita para os analistas e o questionário de avaliação foram encaminhados também para quatro técnicos de alguns dos Pontos de Presença (PoPs) da RNP nos estados brasileiros, os quais estão participando do desenvolvimento do protótipo de rede híbrida da RNP. Tal ação foi baseada na técnica Delphi [Rowe, 2001 e Green et al., 2007], que é um método sistemático e interativo de estimativa que se baseia na experiência independente de especialistas. Tanto os analistas da Gerência de Operações quanto os técnicos dos PoPs da RNP foram selecionados para participar da pesquisa por serem profissionais com experiência comprovada em administração e operação de redes, além de estarem envolvidos com o desenvolvimento de redes híbridas, permitindo assim uma avaliação dos processos adaptados que indicasse ou não se os mesmos são aplicáveis em um ambiente real de operação de redes.

Para cada sub-processo, um grupo de questões foi elaborada com o intuito de avaliar: aplicabilidade do sub-processo adaptado no gerenciamento de uma rede híbrida; aplicabilidade do sub-processo adaptado em um ambiente real de NOC; e impacto do sub-processo adaptado na disponibilização do serviço de aprovisionamento para os seus usuários. A Tabela 2 ilustra de forma consolidada os resultados da avaliação destes itens. São apresentados os resultados da avaliação de cada item, contendo os seus respectivos percentuais, calculados a partir da média simples das respostas dos analistas às questões que, juntas, avaliam um dado item. As siglas DT, DP, NSR, CP e CT 
significam, "Discordo Totalmente", "Discordo Parcialmente", "Não Sei Responder", "Concordo Parcialmente" e "Concordo Totalmente".

Com base nos indicadores apresentados na Tabela 2, observou-se a boa aceitação de todos os sub-processos por parte dos especialistas, o que mostra indícios que os mesmos podem ser aplicados em um ambiente real de operação de redes. Os profissionais que trabalham no dia-a-dia dentro de um ambiente de NOC distinguiram os sub-processos, com as suas adaptações, como sendo aplicáveis no seu ambiente de trabalho, então estes sub-processos podem ser, de fato, utilizados na operação de uma DCN.

\begin{tabular}{|c|c|c|c|c|c|c|}
\hline \multirow[b]{2}{*}{ Sub-processo } & \multicolumn{6}{|c|}{ Questões } \\
\hline & \multicolumn{2}{|c|}{$\begin{array}{c}\text { Aplicabilidade do sub-processo no } \\
\text { gerenciamento de uma rede } \\
\text { híbrida }\end{array}$} & \multicolumn{2}{|c|}{$\begin{array}{c}\text { Aplicabilidade do sub-processo no } \\
\text { ambiente de um NOC }\end{array}$} & \multicolumn{2}{|c|}{$\begin{array}{c}\text { Impacto do sub-processo na } \\
\text { disponibilização do serviço para os } \\
\text { usuários }\end{array}$} \\
\hline \multirow{5}{*}{$\begin{array}{l}\text { Gestão de } \\
\text { Configuração }\end{array}$} & DT & $0 \%$ & DT & $0 \%$ & DT & $0 \%$ \\
\hline & DP & $0 \%$ & DP & $0 \%$ & DP & $25 \%$ \\
\hline & NSR & $0 \%$ & NSR & $0 \%$ & NSR & $0 \%$ \\
\hline & $\mathrm{CP}$ & $13 \%$ & $\mathrm{CP}$ & $0 \%$ & $\mathrm{CP}$ & $38 \%$ \\
\hline & $\mathrm{CT}$ & $88 \%$ & CT & $100 \%$ & CT & $38 \%$ \\
\hline \multirow{5}{*}{$\begin{array}{c}\text { Gestão do } \\
\text { Desempenho }\end{array}$} & DT & $3 \%$ & DT & $0 \%$ & DT & $0 \%$ \\
\hline & $\mathrm{DP}$ & $0 \%$ & $\mathrm{DP}$ & $0 \%$ & DP & $25 \%$ \\
\hline & NSR & $0 \%$ & NSR & $0 \%$ & NSR & $0 \%$ \\
\hline & $\mathrm{CP}$ & $15 \%$ & $\mathrm{CP}$ & $0 \%$ & $\mathrm{CP}$ & $25 \%$ \\
\hline & $\mathrm{CT}$ & $83 \%$ & $\mathrm{CT}$ & $100 \%$ & CT & $50 \%$ \\
\hline \multirow{5}{*}{ Gestão de Falhas } & DT & $0 \%$ & DT & $0 \%$ & DT & $0 \%$ \\
\hline & $\mathrm{DP}$ & $0 \%$ & $\mathrm{DP}$ & $0 \%$ & $\mathrm{DP}$ & $25 \%$ \\
\hline & NSR & $0 \%$ & NSR & $0 \%$ & NSR & $0 \%$ \\
\hline & $\mathrm{CP}$ & $13 \%$ & $\mathrm{CP}$ & $13 \%$ & $\mathrm{CP}$ & $25 \%$ \\
\hline & CT & $88 \%$ & CT & $88 \%$ & CT & $50 \%$ \\
\hline
\end{tabular}

Tabela 2: Avaliação consolidada dos sub-processos

Os sub-processos foram encarados pelos especialistas como partes de um todo, ou seja, o valor de cada sub-processo não os torna independentes entre si. Tal afirmação pode ser feita a partir da opinião unânime dos entrevistados: todos os sub-processos auxiliam na obtenção de alta disponibilidade do serviço de aprovisionamento de circuitos virtuais, mas a alta disponibilidade do serviço não deve ser atribuída a apenas um ou mais dos sub-processos, mas sim a todos.

Com relação ao uso dos sub-processos na obtenção de alta disponibilidade do serviço de circuitos virtuais dinâmicos, os especialistas opinaram sobre outro fator: a implantação de uma arquitetura de rede de circuitos virtuais que disponibilize caminhos redundantes para os softwares construtores dos circuitos, garantindo contingência dos mesmos. Tais observações mostram que, os sub-processos não resolvem todo e qualquer problema referente aos circuitos virtuais dinâmicos. Se uma NREN projetar de forma equivocada a sua infraestrutura de circuitos virtuais, não contemplando diferentes caminhos físicos para o aprovisionamento dos circuitos dinâmicos, a aplicação dos subprocessos (tratamento de falha, tratamento de degradação de desempenho, análise das estatísticas de uso do circuito virtual etc) estará limitada pela arquitetura da rede. Logo, os sub-processos são valiosos na obtenção de um serviço de aprovisionamento dinâmico de qualidade, mas não podem ser considerados suficientes para tal fim.

O último ponto importante obtido das respostas das questões objetivas diz respeito ao sub-processo gestão da segurança. Apesar de ter sido considerado pela maioria como aplicável no gerenciamento de redes híbridas, houve especialistas que não 
conseguiram enxergar potenciais incidentes de segurança oriundos do aprovisionamento dinâmico de circuitos. Logo, este sub-processo deve ser reavaliado, se possível após a sua implantação em um NOC, de forma que uma visão mais apurada da sua relevância na operação do serviço de aprovisionamento seja desenvolvida.

\section{Conclusões e Trabalhos Futuros}

A motivação para o estudo sobre gerenciamento de redes híbridas dá-se pelo fato de que, dentro do âmbito das NREN, o estudo sobre circuitos virtuais dinâmicos vem sendo desenvolvido cada vez mais e um desafio permanece: como monitorar circuitos virtuais que são aprovisionados de forma transparente para os respectivos NOC das redes sobre as quais os mesmos são construídos? Trata-se aqui de uma quebra de paradigma, uma vez que, as tecnologias de circuitos de redes de computadores tradicionais eram aplicadas de forma manual, estática, pelos administradores e operadores das redes, com as configurações sendo executadas sob demanda.

Com base neste cenário, estabeleceu-se como objetivo de pesquisa a adaptação de processos de gerenciamento de redes IP tradicionais, respeitando-se os modelos de gerenciamento de redes da ITU-T. A partir de uma revisão bibliográfica dos principais aspectos de gerenciamento de redes, das recomendações da ITU-T e dos estudos sobre redes híbridas em desenvolvimento, e tomando-se como base os processos originalmente escritos pela RNP para gerenciar o backbone acadêmico brasileiro, foram propostas adaptações nestes processos de tal forma que o serviço de aprovisionamento dinâmico de circuitos virtuais fosse contemplado como um serviço gerenciado. Resultados da avaliação realizada com especialistas indicaram que as adaptações proveem uma forma estruturada de gerenciar circuitos virtuais aprovisionados dinamicamente. Entre as contribuições desta pesquisa, destacam-se: a apresentação da abordagem de gestão de processos para a área de gerenciamento de redes; o fomento à discussão sobre o tema gerenciamento de redes híbridas entre especialistas; e as indicações de integração entre ferramentas de aprovisionamento e de monitoramento.

Contudo, é importante ressaltar que o escopo da proposta foi adaptar processos de gerenciamento de redes IP convencionais para contemplar redes híbridas, e não descrever em detalhes as atividades que os compõem, o que é objeto de trabalhos futuros. Além disso, outras NREN devem ser convidadas a participar da avaliação dos processos. Por fim, os processos adaptados precisam ser reavaliados após a sua efetiva implantação em um ambiente real de NOC. Seguindo o ciclo da Gestão de Processos, os processos poderiam então ser otimizados, de forma a torná-los mais eficientes, bem como as indicações de integração entre ferramentas de aprovisionamento de circuitos virtuais dinâmicos e ferramentas de monitoramento de redes devem ser desenvolvidas, com provas de conceito objetivando validar a comunicação destas entre si.

\section{Referências}

De Antoni, et al. (2001) "Grupo focal: Método qualitativo de pesquisa com adolescentes em situação de risco", Arquivos Brasileiros de Psicologia, 53(2), 38-53.

GÉANT2, http://www.geant2.net. Página acessada pela última vez em 26/01/2013.

Green, A.G. (2007) "Methods to Elicit Forecasts from Groups: Delphi and Prediction Markets Compared", Forthcoming in Foresight: Intern. Journal of Applied Forecasting. 
Gudgin, M., Hadley, M, Mendelsohn, N., Moereau, J.J., Nielsen, H.Frystyk, K., A., Lafon, Y. (2007) "SOAP Version 1.2 Part 1: Messaging Framework (Second Edition)", W3C Recommendation.

Iervolino, SA., Pelicioni, MCF (2001) "A utilização do grupo focal como metodologia qualitativa na promoção da saúde”. Rev Esc Enf USP, v. 35, n.2, p.115-21.

INTERNET2, http://www.internet2.edu/about/. Último acesso: 26/01/2013.

Kurose, J.F., Ross, K. W. (2006) Redes de computadores e a Internet : uma abordagem top-down. $3^{\mathrm{a}}$ edição. São Paulo, Pearson Addison Wesley.

Lake, A., et al. (2008) "Inter-domain Controller (IDC) Protocol Specification".

M.3050.1, ITU-T Recommendation - Enhanced Telecom Operations Map (eTOM) The business process framework. (2007) In: ITU-T Series M: Telecommunication Management, Including TMN and Network Maintenance.

M.3050.2, ITU-T Recommendation - Enhanced Telecom Operations Map (eTOM) Process decompositions and descriptions. (2007) In: ITU-T Series M: Telecommunication Management, Including TMN and Network Maintenance.

M.3400, ITU-T Recommendation - TMN management functions. (2000) In: ITU-T Series M: Telecommunication Management, Including TMN and Network Maintenance.

Naone, E. (2008) "Bandwidth on Demand", MIT Technology Review.

Ostroski, E. H., Digiampietri, L. A., Prado, E. P. V., Albuquerque, J. P., Frazão, J. B., Errante, P. R., Condino-Neto, A. (2012) "Avaliação de BPMN para a caracterização estratégica de uma organização de pesquisas acadêmicas”. In VIII Simpósio Brasileiro de Sistemas de Informação, pages 210-221.

RNP, http://www.rnp.br/rnp/. Último acesso: 26/01/2013.

Rowe, G., Wright, G. (2001) Expert opinions in forecasting: The role of the Delphi technique. In Armstrong, J. S. (Ed.): Principles of forecasting: a handbook for researchers and practitioners, Kluwer Academic Publishers, 125-144, Norwell, MA.

SE-CIPÓ, http://wiki.rnp.br/display/secipo/Home. Último acesso: 26/01/2013.

Weske, M. (2012) Bussiness Process Management: Concepts, Languages, Architectures. Second Edition. Springer. 